\title{
Quantum correction factors for multiphonon processes in condensed phase vibrational relaxation
}

\author{
Binny J. Cherayil \\ Department of Inorganic and Physical Chemistry, Indian Institute of Science, Bangalore-560012, India
}

\begin{abstract}
An influence functional approach is used to determine the role of multiphonon processes in the rates of vibrational relaxation. Relaxation is considered to occur between a pair of coupled harmonic oscillators, representing an excited and a receiving mode on a single polyatomic solute, and a collection of independent harmonic oscillators, representing a solvent reservoir. The interaction between the oscillator pair in the solute is arbitrary and left unspecified, while interactions between solute and solvent are taken to be linear in the solute coordinates but quadratic or cubic in the solvent coordinates. The nonlinearities allow vibrational relaxation to occur through multiple excitations of phonons. Transitions rates for such multiphonon processes are derived, as are quantum corrections to the corresponding classical force correlation functions. The quantum correction factors are also shown to emerge directly from certain terms in the real part of the influence functional.
\end{abstract}

\section{INTRODUCTION}

Calculations of the relaxation rates of vibrationally excited solutes in liquids or other dense media often treat the solute quantum mechanically and the solvent classically whenever a fully quantum mechanical approach is impractical. Although this semi-classical approximation is not always accurate, especially at low temperatures, where the inherent quantum mechanical nature of the system tends to be most strongly manifested, its reliability can be improved by the application of a so-called quantum correction factor (qcf). ${ }^{1-10}$ For vibrational modes that are coupled linearly (in the bath coordinates) to a harmonic bath, this factor can be determined exactly. ${ }^{1}$ The linear coupling case is, however, limited as a description of relaxation involving, say, high frequency vibrational modes, where the process is typically accompanied by the excitation of more than one normal mode (phonon) of the solvent, and is induced by nonlinear couplings between solute and solvent. If the relaxation rate for a process dominated by multiphonon excitation is derived from a classical description of the solvent, the quantum correction factor can no longer be calculated exactly. Unfortunately, even the available approximate correction factors ${ }^{1-10}$ are not all mutually consistent, reflecting the apparent lack of systematic and well-defined methods to calculate them. The goal of the present article is therefore to develop such methods from general principles.

Our approach is based on the influence functional formalism of Feynman and Vernon, ${ }^{11}$ which was very recently used by Shiga and Okazaki ${ }^{12}$ to address precisely this issue of multiphonon relaxation in the condensed phase, including the question of quantum corrections to classical rate expressions. However, no attempt was made in this latter work to actually calculate qcf's, since it was felt that in the absence of a well-motivated approximation scheme, the calculation was unlikely to be useful. This may or may not be the case, but we nevertheless explore the implications of an approxi- mation applied by Egorov and Skinner ${ }^{13}$ to solids that Shiga and Okazaki considered of doubtful relevance to liquids, and so ignored. The calculations of Shiga and Okazaki were also concerned only with the problem of a single solute oscillator in a bath of solvent oscillators, and thus were effectively constrained to model the relaxation of diatomics alone. But a number of recent experiments have been able to probe the vibrational dynamics of polyatomic solutes in polyatomic solvents. ${ }^{14}$ For such systems the foregoing influence functional approach must be generalized, and a very simple generalization is considered here: it is to treat the dynamics of a pair of coupled solute oscillators, one representing the vibrational mode that is excited, and the other representing a mode on the same molecule into which a fraction of the excess energy may be deposited, the rest of the energy being dissipated to the bath through phonon excitation. In this way, one captures the rudiments of the intramolecular relaxation pathways that may be additionally available to polyatomics but not to diatomics. The overall approach in these calculations thus follows the methodolgy of Ref. 12 (which in turn invokes the work described in Ref. 15 on quantum field theory), but the inclusion of a second internal mode in the calculations is a sufficient complication that separate treatment is warranted. For this reason, too, the calculations are presented in sufficient detail that they can be read on their own.

Despite their utility in problems that deal broadly with the general phenomenon of "decoherence," ${ }^{15-17}$ functional integral techniques have not been widely used to study vibrational relaxation. For the most part, they are not needed if the results of low-order perturbation theory provide an adequate first approximation. ${ }^{18}$ The great advantage of the functional integral approach is that it is well-suited for nonperturbative calculations, ${ }^{11}$ but this advantage is not exploited in the present calculation. Shiga and Okazaki have already indicated the directions along which a nonperturba- 
tive calculation of the vibrational relaxation rate could be expected to proceed. ${ }^{12}$ There is, however, another reason for using this approach: it is formulated around the density matrix, which is a convenient starting point for treating condensed phase vibrational dephasing, ${ }^{19,20}$ a subject that will be taken up in future publications.

A review of the approach is presented in the following section; it is elaborated around a model of a pair of coupled harmonic oscillators (representing the polyatomic) that interacts nonlinearly with a collection of independent harmonic oscillators (representing the normal modes of the bath). The spectral properties of the bath are contained in a quantity called the influence functional. As its name suggests, the influence functional determines the extent to which the properties of the bath influence the dynamics of the excited state solute. As such, it is the key element of the present analysis, and its calculation is therefore central to the objectives outlined earlier. However, it is not an easy object to calculate, and we do so only approximately, following the approach originally introduced by $\mathrm{Hu}$ et al. ${ }^{15}$ and adopted later by Shiga and Okazaki. ${ }^{12}$ The formalism is slightly generalized here to treat two coupled oscillators in a condensed phase environment. Although the calculations are relatively straightforward, they are sufficiently lengthy that they are relegated to two mathematical appendices so as not to obscure the main threads of the theoretical development, which are presented in Sec. III, where state-to-state transition rates are calculated along the lines discussed by Feynman and Hibbs. ${ }^{11}$ These results are then used in Sec. IV to derive expressions for the quantum correction factors, which are the main results of the article. A brief comparison of our expressions with others in the literature is presented in Sec. V, which also presents some broad conclusions.

\section{BACKGROUND AND REVIEW}

As a prototype of a polyatomic solute in a solvent, we consider a pair of coupled harmonic oscillators interacting with a bath of $N$ independent harmonic oscillators. The coupled oscillator pair represents both the high frequency mode that is initially excited on the polyatomic by, say, a pump pulse, and a nearby mode on the same molecule (the receiving mode) that receives some fraction of the energy dissipated during the course of relaxation. The coupling between the two is arbitrary, and is not specified. The $N$ independent oscillators represent the modes of the bath, which receive the remainder of the vibrational energy. The Hamiltonian $H$ for such a system is given by

$$
H=H_{X}+H_{B}+H_{X B} \equiv\left(H_{1}+H_{2}+H_{12}\right)+H_{B}+H_{X B},
$$

where

$$
\begin{aligned}
& H_{\alpha}=\frac{1}{2} m_{\alpha} \dot{x}_{\alpha}^{2}+\frac{1}{2} m_{\alpha} \omega_{\alpha}^{2} x_{\alpha}^{2}, \quad \alpha=1,2 \\
& H_{12}=\lambda G\left(x_{1}, x_{2}\right), \\
& H_{B}=\frac{1}{2} \sum_{k=1}^{N} M_{k} \dot{R}_{k}^{2}+\frac{1}{2} \sum_{k=1}^{N} M_{k} \Omega_{k}^{2} R_{k}^{2},
\end{aligned}
$$

and

$$
H_{X B}=A^{(n)} x_{1} q^{n}+B^{(n)} x_{2} q^{n}, \quad n=1,2,3 \ldots .
$$

Here, $x_{1}, x_{2}$ and $R_{k}$, and $\dot{x}_{1}, \dot{x}_{2}$ and $\dot{R}_{k}$ are the displacements and velocities of, respectively, the excited mode, the receiving mode and the $k$ th normal mode of the bath (the symbols are understood to refer to three-dimensional vectors); $m_{1}$ and $m_{2}$ are the reduced masses of the coupled oscillator pair, and $\omega_{1}$ and $\omega_{2}$ are their vibrational frequencies; the corresponding upper case symbols refer to the parameters of the bath; $\lambda$ is the coupling constant between the oscillator pair, and $G\left(x_{1}, x_{2}\right)$ is some arbitrary function of their coordinates; $q$ is a collective coordinate of the bath that is some linear combination of its normal modes, and is defined as

$$
q=\sum_{k=1}^{N} c_{k} R_{k},
$$

where the $c_{k}$ are unknown expansion coefficients; and $A^{(n)}$ and $B^{(n)}$ are the coupling constants between the solute modes and the collective coordinate of the bath. The interaction term in Eq. (5) is a generalization of the nonlinearities studied in Ref. 15, where the analog of $H_{X B}$ was given by $\Sigma_{k} A^{(n)} x_{1} R_{k}$. In condensed phases, especially solids, it is the variable $q$ rather than the normal mode displacement $R_{k}$ that is expected to be the relevant descriptor of collective excitations. $^{13}$

The influence functional approach begins with the following expression for the density operator $\rho(t)$ at time $t::^{11,21}$

$$
\rho(t)=\mathrm{e}^{-i H t / \hbar} \rho(0) \mathrm{e}^{i H t / \hbar} .
$$

Using the definition $\left\langle x_{1}, x_{2}, R|\rho(t)| y_{1}, y_{2}, Q\right\rangle$ $=\rho\left(x_{1}, y_{1} ; x_{2}, y_{2} ; R, Q ; t\right)$ in the coordinate representation, where $x_{1}$ and $y_{1}$ are the displacement coordinates of oscillator $1, x_{2}$ and $y_{2}$ are coordinates of oscillator 2 , and $R$ and $Q$ stand for the coordinates of the bath modes (i.e., $R$ $\equiv\left\{R_{1}, \ldots R_{N}\right\}$ and $\left.Q \equiv\left\{Q_{1}, \ldots Q_{N}\right\}\right)$, it can be shown that

$$
\begin{aligned}
& \rho\left(x_{1}, y_{1} ; x_{2}, y_{2} ; R, Q ; t\right) \\
& =\int d x_{1}^{\prime} d y_{1}^{\prime} d x_{2}^{\prime} d y_{2}^{\prime} d R^{\prime} d Q^{\prime} K\left(x_{1}, x_{2}, R, t ; x_{1}^{\prime}, x_{2}^{\prime}, R^{\prime}, 0\right) \\
& \quad \times \rho\left(x_{1}^{\prime}, y_{1}^{\prime} ; x_{2}^{\prime}, y_{2}^{\prime} ; R^{\prime}, Q^{\prime}\right) K^{*}\left(y_{1}, y_{2}, Q, t ; y_{1}^{\prime}, y_{2}^{\prime}, Q^{\prime}\right),
\end{aligned}
$$

where, in terms of path integrals, the propagator $K\left(x_{1}, x_{2}, R, t ; x_{1}^{\prime}, x_{2}^{\prime}, R^{\prime}\right)$ is given by

$$
\begin{aligned}
& K\left(x_{1}, x_{2}, R, t ; x_{1}^{\prime}, x_{2}^{\prime}, R^{\prime}\right) \\
& =\int_{x_{1}^{\prime}}^{x_{1}} \mathcal{D}\left[x_{1}(t)\right] \int_{x_{2}^{\prime}}^{x_{2}} \mathcal{D}\left[x_{2}(t)\right] \int_{R^{\prime}}^{R} \mathcal{D}[R(t)] \\
& \quad \times \exp \left[\frac{i}{\hbar}\left\{S\left[x_{1}, x_{2}\right]+S_{B}[R]+S_{I}\left[x_{1}, x_{2}, R\right]\right\}\right]
\end{aligned}
$$

with 


$$
\begin{aligned}
& S\left[x_{1}, x_{2}\right]= \frac{1}{2} m_{1} \int_{0}^{t} d \tau\left[\dot{x}_{1}(\tau)^{2}-\omega_{1}^{2} x_{1}(\tau)^{2}\right] \\
&+\frac{1}{2} m_{2} \int_{0}^{t} d \tau\left[\dot{x}_{2}(\tau)^{2}-\omega_{2}^{2} x_{2}(\tau)^{2}\right] \\
&-\lambda \int_{0}^{t} d \tau G\left(x_{1}(\tau), x_{2}(\tau)\right), \\
& S_{B}[R]=\frac{1}{2} \sum_{k=1}^{N} M_{k} \int_{0}^{t} d \tau\left[\dot{R}_{k}(\tau)^{2}-\Omega_{k}^{2} R_{k}^{2}(\tau)\right],
\end{aligned}
$$

and

$$
\begin{aligned}
S_{I}\left[x_{1}, x_{2}, R\right]= & -A^{(n)} \int_{0}^{t} d \tau x_{1}(\tau) q(\tau)^{n} \\
& -B^{(n)} \int_{0}^{t} d \tau x_{2}(\tau) q(\tau)^{n}, \quad n=1,2,3 \ldots
\end{aligned}
$$

The propagator $K^{*}\left(y_{1}, y_{2}, Q, t ; y_{1}^{\prime}, y_{2}^{\prime}, Q^{\prime}\right)$ is the complex conjugate of the propagator $K\left(x_{1}, x_{2}, R, t ; x_{1}^{\prime}, x_{2}^{\prime}, R^{\prime}\right)$ with the $\{x\}$ coordinates replaced by $\{y\}$ coordinates and the $R$ coordinates by $Q$ coordinates. Here $\mathcal{D}\left[x_{1}(t)\right]$ represents the functional measure on the space of $x_{1}$ trajectories, and the limits $x_{1}^{\prime}$ and $x_{1}$ define the end-point conditions $x_{1}(0)=x_{1}^{\prime}$ and $x_{1}(t)=x_{1}$. The path integrals over $x_{2}$ and $R$, and over $y_{1}, y_{2}$ and $Q$ are defined similarly.

Defining a bath averaged density of states as

$$
\widetilde{\rho}\left(x_{1}, y_{1} ; x_{2}, y_{2} ; t\right) \equiv \int d R \rho\left(x_{1}, y_{1} ; x_{2}, y_{2} ; R, R ; t\right)
$$

and assuming separability of the initial equilibrium density matrix, which implies

$$
\rho\left(x_{1}^{\prime}, y_{1}^{\prime} ; x_{2}^{\prime}, y_{2}^{\prime} ; R^{\prime}, Q^{\prime}\right)=\rho\left(x_{1}^{\prime}, y_{1}^{\prime} ; x_{2}^{\prime}, y_{2}^{\prime}\right) \rho_{B}\left(R^{\prime}, Q^{\prime}\right),
$$

it is readily shown that

$$
\begin{aligned}
\widetilde{\rho}\left(x_{1}, y_{1} ; x_{2}, y_{2} ; t\right) \\
=\int d x_{1}^{\prime} d y_{1}^{\prime} d x_{2}^{\prime} d y_{2}^{\prime} \int_{x_{1}^{\prime}}^{x_{1}} \mathcal{D}\left[x_{1}(t)\right] \int_{y_{1}^{\prime}}^{y_{1}} \mathcal{D}\left[y_{1}(t)\right] \\
\quad \times \int_{x_{2}^{\prime}}^{x_{2}} \mathcal{D}\left[x_{2}(t)\right] \int_{y_{2}^{\prime}}^{y_{2}} \mathcal{D}\left[y_{2}(t)\right] \exp \left[\frac { i } { \hbar } \left\{S\left[x_{1}, x_{2}\right]\right.\right. \\
\left.\left.\quad-S\left[y_{1}, y_{2}\right]\right\}\right] F\left[x_{1}, y_{1} ; x_{2}, y_{2}\right] \rho\left(x_{1}^{\prime}, y_{1}^{\prime} ; x_{2}^{\prime}, y_{2}^{\prime}\right),
\end{aligned}
$$

where the quantity $F\left[x_{1}, y_{1} ; x_{2}, y_{2}\right]$-the influence functional-is given by

$$
\begin{aligned}
& F\left[x_{1}, y_{1} ; x_{2}, y_{2}\right] \\
& =\int d R^{\prime} d Q^{\prime} d R \int_{R^{\prime}}^{R} \mathcal{D}[R(t)] \int_{Q^{\prime}}^{R} \mathcal{D}[Q(t)] \exp \left[\frac { i } { \hbar } \left\{S_{B}[R]\right.\right. \\
& \left.\left.\quad-S_{B}[Q]+S_{I}\left[x_{1}, x_{2}, R\right]-S_{I}\left[y_{1}, y_{2}, Q\right]\right\}\right] \rho_{B}\left(R^{\prime}, Q^{\prime}\right)
\end{aligned}
$$

All of the information relevant to condensed phase vibrational relaxation is contained in the influence functional $F$, which describes the extent to which the presence of solvent modifies the dynamical behavior of the isolated oscillator pair. When there is no coupling whatever between solute and solvent, $F=1$. Departures of $F$ from unity indicate the involvement of the solvent in the rate of transition between different eigenstates of the unperturbed solute.

At present, the influence functional can be calculated exactly only when the coupling between the solute and solvent is linear in their respective coordinates. When the interactions between them involve nonlinearities, $F$ can no longer be calculated exactly, but it can be obtained approximately by means of a cumulant expansion around the exact bilinear coupling results, as first suggested by $\mathrm{Hu}$ et $a l .{ }^{15}$ in the context of quantum field theory. The procedure is rigorous and systematic, and can be carried through to arbitrary order of approximation in the cumulant expansion. The details of the calculation, though important, are largely standard, so they are not discussed in the main part of the article, but they can be found in the appendices. The following section makes use of the results presented there to calculate transition rates between different eigenstates of the oscillator pair.

\section{TRANSITION RATES}

If it is assumed that the system (oscillators 1 and 2) is initially in the state characterized by some set of quantum numbers collectively denoted $I$ (this state being the eigenstate of the unperturbed system), and if it is further assumed that the state is occupied with probability 1 to reflect typical initial conditions in pump-probe experiments, then given the influence functional, one may calculate the probability that the system then makes a transition to a final state $|N\rangle$ in the time interval $t$. The initial condition is expressed as

$$
\rho(0)=|I\rangle\langle I| \text {. }
$$

Thus in coordinate representation,

$$
\begin{aligned}
\rho\left(x_{1}^{\prime}, y_{1}^{\prime} ; x_{2}^{\prime}, y_{2}^{\prime}\right) & =\left\langle x_{1}^{\prime}, x_{2}^{\prime}|\rho(0)| y_{1}^{\prime}, y_{2}^{\prime}\right\rangle \\
& =\left\langle x_{1}^{\prime}, x_{2}^{\prime} \mid I\right\rangle\left\langle I \mid y_{1}^{\prime}, y_{2}^{\prime}\right\rangle \\
& =\psi_{I}\left(x_{1}^{\prime}, x_{2}^{\prime}\right) \psi_{I}^{*}\left(y_{1}^{\prime}, y_{2}^{\prime}\right),
\end{aligned}
$$

where $\psi_{I}\left(x_{1}^{\prime}, x_{2}^{\prime}\right)$ is the solution to the Schrödinger equation for the coupled oscillators in the absence of solvent [which is in general unknown unless $G\left(x_{1}, x_{2}\right)$ happens to be bilinear in $x_{1}$ and $x_{2}$.] The probability $P_{N}(t)$ that the system is in the state $|N\rangle$ at time $t$ is

$$
P_{N}(t)=\langle N|\widetilde{\rho}(t)| N\rangle,
$$


which, introducing complete sets of states, can be written as

$$
\begin{aligned}
P_{N}(t)= & \int d x_{1} d x_{2} d y_{1} d y_{2} \psi_{N}^{*}\left(x_{1}, x_{2}\right) \\
& \times \widetilde{\rho}\left(x_{1}, y_{1} ; x_{2}, y_{2} ; t\right) \psi_{N}\left(y_{1}, y_{2}\right) .
\end{aligned}
$$

Equation (15) defining $\widetilde{\rho}\left(x_{1}, y_{1} ; x_{2}, y_{2} ; t\right)$ is substituted into Eq. (20), and as in Feynman and Hibbs, ${ }^{11}$ the influence functional is then linearized by expanding to first order in the function $\alpha_{j}$, which is related to the mean thermal occupation number, and is defined in Eqs. (A7)-(A9). (This procedure is analogous to the conventional perturbation approach based on the Schrödinger equation.) Each term in the expansion (including the leading order term of unity) is the product of two factors, each of which is of the general form

$$
\begin{gathered}
\int d x_{1} d x_{2} d x_{1}^{\prime} d x_{2}^{\prime} \int_{x_{1}^{\prime}}^{x_{1}} \mathcal{D}\left[x_{1}\right] \int_{x_{2}^{\prime}}^{x_{2}} \mathcal{D}\left[x_{2}\right] \\
\times \chi^{*}\left(x_{1}, x_{2}\right) A \mathrm{e}^{i S / \hbar} \xi\left(x_{1}^{\prime}, x_{2}^{\prime}\right)
\end{gathered}
$$

where $A$ is some functional of the oscillator coordinates. This expression defines - in the notation of Feynman and Hibbs-the transition element $\langle\chi|A| \xi\rangle_{S}$. (The sign of $S$ in the above equation may be positive or negative; the transition element with negative $S$ is the complex conjugate of the transition element with positive $S$.) When $A$ is unity, the transition element vanishes by virtue of the orthonormality of the eigenfunctions of the isolated coupled oscillator problem. For other values of $A$, the transition element may be expressed in terms of various overlap integrals, using the general results,

$$
\left\langle N\left|x_{\alpha}(\tau)\right| I\right\rangle \equiv x_{N I}^{(\alpha)} \mathrm{e}^{-i\left(E_{N}-E_{I}\right) \tau / \hbar}, \quad \alpha=1,2,
$$

and

$$
\left\langle I\left|y_{\alpha}\left(\tau^{\prime}\right)\right| N\right\rangle^{*} \equiv y_{N I}^{(\alpha)} \mathrm{e}^{i\left(E_{N}-E_{I}\right) \tau^{\prime} / \hbar}, \quad \alpha=1,2,
$$

where

$$
x_{N I}^{(\alpha)} \equiv \int d x_{1} d x_{2} \psi_{N}^{*}\left(x_{1}, x_{2}\right) x_{\alpha} \psi_{I}\left(x_{1}, x_{2}\right), \quad \alpha=1,2
$$

$P_{N}(t)$ is calculated in this way for different values of $n$, the number of phonons involved in the process. For $n \geqslant 2$, the probability is denoted $P_{N}^{(n)}(t) . P_{N}^{(2)}(t)$ and $P_{N}^{(3)}(t)$ are given in particular by

$$
\begin{aligned}
P_{N}^{(2)}(t)= & \sum_{m=1}^{N} \sum_{n=1}^{N} \frac{\gamma_{m n}^{2}}{4 M_{m} M_{n} \Omega_{m} \Omega_{n}} \int_{0}^{t} d \tau \int_{0}^{t} d \tau^{\prime}\left[\alpha_{m}\left(\tau-\tau^{\prime}\right)\right. \\
& \times \alpha_{n}\left(\tau-\tau^{\prime}\right) \mathrm{e}^{-i \omega_{0}\left(\tau-\tau^{\prime}\right)}+\alpha_{m}^{*}\left(\tau-\tau^{\prime}\right) \alpha_{n}^{*}\left(\tau-\tau^{\prime}\right) \\
& \left.\times \mathrm{e}^{i \omega_{0}\left(\tau-\tau^{\prime}\right)}\right] .
\end{aligned}
$$

Here $\hbar \omega_{0}$ is the energy difference $E_{I}-E_{N}$ between the initial and final states of the coupled oscillator pair; the functions $\alpha_{i}\left(\tau-\tau^{\prime}\right)$, as stated before, are defined in Eqs. (A7)-(A9); and the parameter $\gamma_{m n}$ is related to the coupling constants between the solute oscillators and the collective coordinate of the bath, and is defined by

$$
\begin{aligned}
\gamma_{m n}^{2}= & c_{m}^{2} c_{n}^{2}\left[\left(A^{(2)} x_{N I}^{(1)}\right)^{2}+\left(B^{(2)} x_{N I}^{(2)}\right)^{2}\right. \\
& \left.+2 A^{(2)} B^{(2)} x_{N I}^{(1)} x_{N I}^{(2)}\right] .
\end{aligned}
$$

Similarly,

$$
\begin{aligned}
P_{N}^{(3)}(t)= & \frac{3 \hbar}{32} \sum_{l, m, n} \frac{\sigma_{l m n}^{2}}{M_{l} M_{m} M_{n} \Omega_{l} \Omega_{m} \Omega_{n}} \int_{0}^{t} \int_{0}^{t} d \tau d \tau^{\prime} \\
& \times\left\{\left[4 \alpha_{l}\left(\tau-\tau^{\prime}\right) \alpha_{m}\left(\tau-\tau^{\prime}\right) \alpha_{n}\left(\tau-\tau^{\prime}\right)\right.\right. \\
& \left.+6 \alpha_{l}(0) \alpha_{m}(0) \alpha_{n}\left(\tau-\tau^{\prime}\right)\right] \mathrm{e}^{-i \omega_{0}\left(\tau-\tau^{\prime}\right)} \\
& +\left[4 \alpha_{l}^{*}\left(\tau-\tau^{\prime}\right) \alpha_{m}^{*}\left(\tau-\tau^{\prime}\right) \alpha_{n}^{*}\left(\tau-\tau^{\prime}\right)\right. \\
& \left.\left.+6 \alpha_{l}(0) \alpha_{m}(0) \alpha_{n}^{*}\left(\tau-\tau^{\prime}\right)\right] \mathrm{e}^{i \omega_{0}\left(\tau-\tau^{\prime}\right)}\right\}
\end{aligned}
$$

where

$$
\begin{aligned}
\sigma_{l m n}^{2} \equiv & c_{l}^{2} c_{m}^{2} c_{n}^{2}\left[\left(A^{(3)} x_{N I}^{(1)}\right)^{2}+\left(B^{(3)} x_{N I}^{(2)}\right)^{2}\right. \\
& \left.+2 A^{(3)} B^{(3)} x_{N I}^{(1)} x_{N I}^{(2)}\right] .
\end{aligned}
$$

If now the rate of transition $k^{(n)}$ between the two states $|I\rangle$ and $|N\rangle$ is defined as

$$
k^{(n)}=\lim _{t \rightarrow \infty} \frac{1}{t} P_{N}^{(n)}(t)
$$

it is readily shown that

$$
k^{(2)}=\operatorname{Re} \sum_{m} \sum_{n} \frac{\gamma_{m n}^{2}}{M_{m} M_{n} \Omega_{m} \Omega_{n}} \int_{0}^{\infty} d t \alpha_{m}(t) \alpha_{n}(t) \mathrm{e}^{-i \omega_{0} t},
$$

where Re denotes real part. When Eq. (A9) is substituted into the above expression, and the resulting integral evaluated according to ${ }^{11}$

$$
\int_{0}^{\infty} d t \mathrm{e}^{ \pm i(\omega \pm \nu) t}= \pm i \mathrm{PP} \frac{1}{\omega \pm \nu}+\pi \delta(\omega \pm \nu)
$$

where PP stands for principal part, it is found that

$$
\begin{aligned}
k^{(2)}= & \pi \sum_{m} \sum_{n} \frac{\gamma_{m n}^{2}}{M_{m} M_{n} \Omega_{m} \Omega_{n}}\left[( 1 + n _ { m } ) ( 1 + n _ { n } ) \delta \left(\omega_{0}\right.\right. \\
& \left.-\left(\Omega_{m}+\Omega_{n}\right)\right)+n_{m}\left(1+n_{n}\right) \delta\left(\omega_{0}-\left(\Omega_{n}-\Omega_{m}\right)\right) \\
& +n_{n}\left(1+n_{m}\right) \delta\left(\omega_{0}-\left(\Omega_{m}-\Omega_{n}\right)\right)+n_{n} n_{m} \delta\left(\omega_{0}\right. \\
& \left.\left.+\left(\Omega_{m}+\Omega_{n}\right)\right)\right]
\end{aligned}
$$

where $n_{k} \equiv 1 /\left[\exp \left(\beta \hbar \Omega_{k}\right)-1\right]$, with $\beta=1 / k_{B} T$.

The transition rate $k^{(3)}$ is similarly found to be 


$$
\begin{aligned}
k^{(3)}= & \frac{3 \pi \hbar}{2} \sum_{l, m, n}\left\{\frac { \sigma _ { l m n } ^ { 2 } } { M _ { l } M _ { m } M _ { n } \Omega _ { l } \Omega _ { m } \Omega _ { n } } \left[\left(1+n_{l}\right)\left(1+n_{m}\right)\left(1+n_{n}\right) \delta\left(\omega_{o}-\left(\Omega_{l}+\Omega_{m}+\Omega_{n}\right)\right)\right.\right. \\
& +n_{l}\left(1+n_{m}\right)\left(1+n_{n}\right) \delta\left(\omega_{o}-\left(\Omega_{m}+\Omega_{n}-\Omega_{l}\right)\right)+n_{m}\left(1+n_{l}\right)\left(1+n_{n}\right) \delta\left(\omega_{o}-\left(\Omega_{l}+\Omega_{n}-\Omega_{m}\right)\right) \\
& +n_{n}\left(1+n_{l}\right)\left(1+n_{m}\right) \delta\left(\omega_{o}-\left(\Omega_{l}+\Omega_{m}-\Omega_{n}\right)\right)+n_{l} n_{m}\left(1+n_{n}\right) \delta\left(\omega_{o}-\left(\Omega_{n}-\Omega_{l}-\Omega_{m}\right)\right) \\
& +n_{l} n_{n}\left(1+n_{m}\right) \delta\left(\omega_{o}-\left(\Omega_{m}-\Omega_{l}-\Omega_{n}\right)\right)+n_{m} n_{n}\left(1+n_{l}\right) \delta\left(\omega_{o}-\left(\Omega_{l}-\Omega_{m}-\Omega_{n}\right)\right) \\
& \left.\left.\left.+n_{l} n_{m} n_{n} \delta\left(\omega_{o}+\Omega_{l}+\Omega_{m}+\Omega_{n}\right)\right)+\frac{3}{2}\left(1+2 n_{l}\right)\left(1+2 n_{m}\right)\left\{\left(1+n_{n}\right) \delta\left(\omega_{0}-\Omega_{n}\right)+n_{n} \delta\left(\omega_{0}+\Omega_{n}\right)\right\}\right]\right\} .
\end{aligned}
$$

\section{CORRECTIONS TO CLASSICAL TRANSITION RATES}

In this section, approximate quantum correction factors are derived for systems in which the interactions between solute and solvent are quadratic or cubic in the solvent collective coordinate. Before turning to these results, however, we provide here, for the sake of completeness, and for purposes of illustration, the expression $k^{(1)}$ for the transition rate between the given initial and final state of a system characterized by bilinear coupling between solute and solvent (which corresponds to a one-phonon process):

$$
\begin{aligned}
k^{(1)}= & \frac{\pi}{\hbar} \sum_{m} \frac{\varepsilon_{m}^{2}}{M_{m} \Omega_{m}}\left[\left(1+n_{m}\right) \delta\left(\omega_{0}-\Omega_{m}\right)\right. \\
& \left.+n_{m} \delta\left(\omega_{0}+\Omega_{m}\right)\right],
\end{aligned}
$$

where

$$
\varepsilon_{m}^{2}=\left(A^{(1)} x_{N I}^{(1)}\right)^{2}+\left(B^{(1)} x_{N I}^{(2)}\right)^{2}+2 A^{(1)} B^{(1)} x_{N I}^{(1)} x_{N I}^{(2)} .
$$

If $\omega_{0}>0$, as we shall assume, only the first term in Eq. (34) survives; moreover, by virtue of the properties of the delta function, the thermal occupation factor may be taken outside the summation sign and evaluated at the frequency $\omega_{o}$ of the transition. If in addition a spectral density function $J^{(1)}(\omega)$ is defined by

$$
J^{(1)}(\omega)=\sum_{m} \frac{\varepsilon_{m}^{2}}{M_{m} \Omega_{m}} \delta\left(\omega-\Omega_{m}\right)
$$

then Eq. (34) reduces to

$$
k^{(1)}=\frac{\pi}{\hbar}\left[1+n\left(\omega_{0}\right)\right] J^{(1)}\left(\omega_{0}\right) .
$$

The general relation between state-to-state transition rates and force correlation functions has been derived by Bader and Berne, ${ }^{1}$ and is given by

$$
k^{(1)}=\frac{2\left|S_{I N}\right|^{2}}{\beta \hbar^{2}\left[1+\mathrm{e}^{-\beta \hbar \omega_{0}}\right]} \widetilde{\zeta}_{\mathrm{qm}}^{\prime}\left(\omega_{0}\right),
$$

where $S_{I N} \equiv\left\langle\psi_{N}^{*}\left(x_{1}, x_{2}\right)\left[A^{(1)} x_{1}+B^{(1)} x_{2}\right] \psi_{I}\left(x_{1}, x_{2}\right)\right\rangle$, and where $\widetilde{\zeta}_{\mathrm{qm}}^{\prime}\left(\omega_{0}\right)$, in the notation of Bader and Berne, is the real part of the Fourier transform of a symmetrized quantum mechanical force correlation function:

$$
\widetilde{\zeta}_{\mathrm{qm}}^{\prime}\left(\omega_{0}\right)=\frac{1}{2 k_{B} T} \operatorname{Re} \int_{0}^{\infty} d t \mathrm{e}^{-i \omega_{0} t}\left\langle[f(t), f(0)]_{+}\right\rangle_{\mathrm{qm}},
$$

$f$ being a force, and the subscript qm denoting quantum mechanical. As mentioned earlier, it is often only the classical friction coefficient $\widetilde{\zeta}_{\mathrm{cl}}^{\prime}\left(\omega_{0}\right)$, and not its quantum counterpart $\widetilde{\zeta}_{\mathrm{qm}}^{\prime}\left(\omega_{0}\right)$, that is known (from molecular dynamics simulations, for example). A correction factor must therefore be applied to bring the two into correspondence. $\widetilde{\zeta}_{\mathrm{cl}}^{\prime}\left(\omega_{0}\right)$ may be derived from the limit $\hbar \rightarrow 0$ of Eq. (38) [together with Eq. (37) defining $\left.k^{(1)}\right]$, which leads to

$$
\begin{aligned}
\widetilde{\zeta}_{\mathrm{cl}}^{\prime}\left(\omega_{0}\right) & =\lim _{\hbar \rightarrow 0} \frac{\pi \beta \hbar}{2\left|S_{I N}\right|^{2}} \operatorname{coth}\left(\beta \hbar \omega_{0} / 2\right) J^{(1)}\left(\omega_{0}\right) \\
& =\frac{\pi}{\omega_{0}\left|S_{I N}(0)\right|^{2}} J^{(1)}\left(\omega_{0}\right),
\end{aligned}
$$

where $S_{I N}(0)$ is the leading order $\hbar \rightarrow 0$ limit of the matrix element $S_{I N}$. From Eqs. (37), (38) and (41), the relation between the quantum and classical friction coefficients is seen to be

$$
\widetilde{\zeta}_{\mathrm{qm}}^{\prime}\left(\omega_{0}\right)=\frac{\left|S_{I N}(0)\right|^{2}}{\left|S_{I N}\right|^{2}} \frac{\hbar \omega_{0}}{2 k_{B} T} \operatorname{coth}\left(\frac{\hbar \omega_{0}}{2 k_{B} T}\right) \widetilde{\zeta}_{\mathrm{cl}}^{\prime}\left(\omega_{0}\right) .
$$

When the system under consideration is a single harmonic oscillator bilinearly coupled to a set of independent harmonic oscillators representing the bath, $S_{I N}=S_{I N}(0)$, and Eq. (42) reduces to the well-known relation between the quantum and classical friction coefficients derived by Bader and Berne. ${ }^{1}$ For a single phonon process, therefore, one may define a quantum correction factor $Q_{1}$ as the ratio of the quantum to the classical frequency-dependent friction coefficients. (This definition leads to somewhat more compact expressions than one defined alternatively in terms of state-to-state transition rates or in terms of the overall population relaxation rate.) Thus,

$$
Q_{1}=\frac{\left|S_{I N}(0)\right|^{2}}{\left|S_{I N}\right|^{2}} \frac{\hbar \omega_{0}}{2 k_{B} T} \operatorname{coth}\left(\frac{\hbar \omega_{0}}{2 k_{B} T}\right)
$$

Correction factors for multiphonon processes are derived in much the same way. For instance, for systems with quadratic coupling between solute and solvent (corresponding to a two-phonon process), the expression for the rate $k^{(2)}$, in the absence of phonon absorption, is from Eq. (32) given by 


$$
\begin{aligned}
k^{(2)}= & \pi \sum_{m, n} \frac{\gamma_{m n}^{2}}{M_{m} M_{n} \Omega_{m} \Omega_{n}}\left(1+n_{m}\right)\left(1+n_{n}\right) \\
& \times \delta\left(\omega_{0}-\Omega_{m}-\Omega_{n}\right) .
\end{aligned}
$$

For reasons explained later, Egorov and Skinner ${ }^{13}$ have argued that in solid-state multiphonon relaxation, the frequency dependence of the thermal occupation factors is much less than that of the degeneracy factor associated with $\gamma_{m n}^{2}$, and that it is therefore a reasonable approximation to take such factors outside the summation sign and to evaluate them at half the frequency of the transition $\omega_{0}$. Adopting this approximation here (essentially $a d h o c$ ), and introducing a new spectral density function $J^{(2)}(\omega)$ defined by

$$
J^{(2)}(\omega)=\sum_{m, n} \frac{\gamma_{m n}^{2}}{M_{m} M_{n} \Omega_{m} \Omega_{n}} \delta\left(\omega-\Omega_{m}-\Omega_{n}\right),
$$

it follows that

$$
k^{(2)} \approx \pi\left[1+n\left(\omega_{0} / 2\right)\right]^{2} J^{(2)}\left(\omega_{0}\right) .
$$

Hence, using $\widetilde{\zeta}_{\mathrm{qm}}^{\prime}\left(\omega_{0}\right)=\beta \hbar^{2}\left[1+\exp \left(-\beta \hbar \omega_{0}\right)\right] k^{(2)} / 2\left|S_{I N}\right|^{2}$, we find

$$
\widetilde{\zeta}_{\mathrm{qm}}^{\prime}\left(\omega_{0}\right)=\frac{\pi \beta \hbar^{2}}{4\left|S_{I N}\right|^{2}}\left[\operatorname{coth}^{2}\left(\beta \hbar \omega_{0} / 4\right)+1\right] J^{(2)}\left(\omega_{0}\right),
$$

the classical limit of which is

$$
\widetilde{\zeta}_{\mathrm{cl}}^{\prime}\left(\omega_{0}\right)=\frac{4 \pi}{\beta \omega_{0}^{2}\left|S_{I N}(0)\right|^{2}} J^{(2)}\left(\omega_{0}\right),
$$

and therefore

$\widetilde{\zeta}_{\mathrm{qm}}^{\prime}\left(\omega_{0}\right)=\frac{\left|S_{I N}(0)\right|^{2}}{\left|S_{I N}\right|^{2}}\left(\frac{\hbar \omega_{0}}{4 k_{B} T}\right)^{2}\left[\operatorname{coth}^{2}\left(\frac{\hbar \omega_{0}}{4 k_{B} T}\right)+1\right] \widetilde{\zeta}_{\mathrm{cl}}^{\prime}\left(\omega_{0}\right)$.

In this two-phonon process, therefore, the quantum correction factor, $Q_{2}$, may be identified as

$$
Q_{2}=\frac{\left|S_{I N}(0)\right|^{2}}{\left|S_{I N}\right|^{2}}\left(\frac{\hbar \omega_{0}}{4 k_{B} T}\right)^{2}\left[\operatorname{coth}^{2}\left(\frac{\hbar \omega_{0}}{4 k_{B} T}\right)+1\right] .
$$

For the case of cubic coupling between solute and solvent, and under similar approximations,

$$
\begin{aligned}
k^{(3)}= & \frac{3 \pi \hbar}{2} \sum_{l, m, n} \frac{\sigma_{l m n}^{2}}{M_{l} M_{m} M_{n} \Omega_{l} \Omega_{m} \Omega_{n}}\left(1+n_{l}\right)\left(1+n_{m}\right) \\
& \times\left(1+n_{n}\right) \delta\left(\omega_{0}-\Omega_{l}-\Omega_{m}-\Omega_{n}\right) \\
\approx & \frac{3 \pi \hbar}{2}\left[1+n\left(\omega_{0} / 3\right)\right]^{3} \sum_{l, m, n} \frac{\sigma_{l m n}^{2}}{M_{l} M_{m} M_{n} \Omega_{l} \Omega_{m} \Omega_{n}} \\
& \times \delta\left(\omega_{0}-\Omega_{l}-\Omega_{m}-\Omega_{n}\right) \\
\equiv & \frac{3 \pi \hbar}{2}\left[1+n\left(\omega_{0} / 3\right)\right]^{3} J^{(3)}\left(\omega_{0}\right) .
\end{aligned}
$$

With $\widetilde{\zeta}_{\text {qm }}^{\prime}\left(\omega_{0}\right)=\beta \hbar^{2}\left[1+\exp \left(-\beta \hbar \omega_{0}\right)\right] k^{(3)} / 2\left|S_{I N}\right|^{2}$, we obtain

$$
\begin{aligned}
\widetilde{\zeta}_{\mathrm{qm}}^{\prime}\left(\omega_{0}\right)= & \frac{3 \pi \beta \hbar^{3}}{16\left|S_{I N}\right|^{2}}\left[\operatorname{coth}^{3}\left(\beta \hbar \omega_{0} / 6\right)\right. \\
& \left.+3 \operatorname{coth}\left(\beta \hbar \omega_{0} / 6\right)\right] J^{(3)}\left(\omega_{0}\right),
\end{aligned}
$$

so that eventually

$$
\begin{aligned}
\widetilde{\zeta}_{\mathrm{qm}}^{\prime}\left(\omega_{0}\right)= & \frac{\left|S_{I N}(0)\right|^{2}}{\left|S_{I N}\right|^{2}}\left(\frac{\hbar \omega_{0}}{6 k_{B} T}\right)^{3}\left[\operatorname{coth}^{3}\left(\frac{\hbar \omega_{0}}{6 k_{B} T}\right)\right. \\
& \left.+3 \operatorname{coth}\left(\frac{\hbar \omega_{0}}{6 k_{B} T}\right)\right] \widetilde{\zeta}_{\mathrm{cl}}^{\prime}\left(\omega_{0}\right) .
\end{aligned}
$$

Thus, the three-phonon quantum correction factor $Q_{3}$ is given by

$Q_{3}=\frac{\left|S_{I N}(0)\right|^{2}}{\left|S_{I N}\right|^{2}}\left(\frac{\hbar \omega_{0}}{6 k_{B} T}\right)^{3}\left[\operatorname{coth}^{3}\left(\frac{\hbar \omega_{0}}{6 k_{B} T}\right)+3 \operatorname{coth}\left(\frac{\hbar \omega_{0}}{6 k_{B} T}\right)\right]$.

Similar results for still higher order processes may be obtained in the same way.

\section{DISCUSSION}

Equations (50) and (54) are the sought for qcf's for quadratic and cubic phonon excitation processes. Expressions for higher order processes may be obtained similarly. The correction factors are, of course, approximate, and are derived by making two key assumptions: (1) that thermal occupation factors, by virtue of being much less frequency dependent than the bath spectral density, may be evaluated at a single frequency, and (2) that this frequency may be determined by dividing the energy deposited into the bath equally among the phonons that are excited. The second of these assumptions ensures that the number of phonons excited during the course of relaxation is as small as possible; such an outcome is generally more likely than one where the number of excited phonons (the order of the process) is larger. These same asumptions have been used with some success in the analysis of multiphonon relaxation in solids, ${ }^{13}$ where they appear to be at least partly justifiable. Their application here to liquid state dynamics is perhaps not as well motivated, but there are few other approximations that are both physically meaningful and analytically tractable. The simplest route to the characterization of multiphonon relaxation rates is through their temperature dependence, so experiments that measure relaxation as a function of temperature may provide direct tests of these approximations.

Other quantum correction factors for multiphonon relaxation are known. For instance, Egorov and Berne ${ }^{3}$ have investigated the vibrational dynamics of an effectively harmonic model of solute and solvent in which the two components have identical normal modes and their mutual interactions are either exponential or power law in the bath coordinates. Their approximate results are found to be in good agreement with exact numerical results obtained by simulation over a fairly wide range of external conditions, provided the spectral density of the bath is assumed to have a particular form (super-ohmic with Gaussian cut-off in the exponential case, and ohmic with Gaussian cut-off in the power law case). From their results for the exponential coupling case, a quantum correction factor $Q_{\mathrm{EB}}$ defined by the relation $\widetilde{\zeta}_{q m}^{\prime}\left(\omega_{0}\right)=Q_{\mathrm{EB}} \widetilde{\zeta}_{c l}^{\prime}\left(\omega_{0}\right)$ can be obtained as

$$
Q_{\mathrm{EB}}=\frac{\exp \left(C_{q m}(0)\right)}{\exp \left(C_{c l}(0)\right)} \cosh \left(\beta \hbar \omega_{0} / 2\right)\left(\frac{\phi_{c l}}{\phi_{q m}}\right)^{\omega_{0} / \omega_{p h}},
$$


where $C_{q m}(0), C_{c l}(0), \phi_{q m}$ and $\phi_{c l}$ are various parameters that characterize the spectral properties of the bath. An analogous but more complicated expression for the correction factor can be obtained for the power law case. In comparisons with exact numerical data, the Egorov-Berne results seem to perform somewhat better than earlier suggested correction factors. ${ }^{4-6}$ But one of these earlier expressions (that of Schofield ${ }^{4}$ ) is notable for being both simple and accurate; it is given by

$$
Q_{S}=\cosh \left(\hbar \omega_{0} / 2 k_{B} T\right) .
$$

The same factor appears in Eq. (55), so the success of $Q_{S}$ in reproducing numerical trends suggests that the other two contributions to the Egorov-Berne formula, $Q_{\mathrm{EB}}$, effectively cancel. Rostkier-Edelstein et al. ${ }^{9}$ have also derived approximate correction factors for multiphonon processes. Defining these factors through $\widetilde{\zeta}_{q m}^{\prime}\left(\omega_{0}\right)=Q_{\mathrm{REGN}} \widetilde{\zeta}_{c l}^{\prime}\left(\omega_{0}\right)$, it can be shown that for a two-phonon process, for example,

$$
Q_{\mathrm{REGN}}=\frac{\beta^{2} \hbar^{2} \omega_{0} \omega_{D}}{4 \tanh \left(\beta \hbar \omega_{0} / 2\right)} \operatorname{coth}\left(\beta \hbar \omega_{D} / 2\right)
$$

where $\omega_{\mathrm{D}}$ is a Debye cut-off frequency. Direct comparisons of the above results with those presented in this article are complicated by the different forms for the solute-solvent coupling and by different sets of approximations.

Apart from its application to the determination of qcf's, the foregoing work is partly intended to lay the foundations for a more extended nonperturbative treatment of vibrational relaxation (and eventually of vibrational dephasing). The present calculations [principally Eqs. (32) and (33)], which employ first order perturbation theory, corroborate the treatment of multiphonon effects discussed by Kenkre et al. ${ }^{22}$ using methods analogous to those used in solid state phonon scattering calculations. They are somewhat more general than these calculations, however, in that the coupled high frequency modes on the solute that are often suspected of being implicated in relaxation are treated explicitly by modeling them as coupled harmonic oscillators.

An important feature of the present functional integral approach is that quantum mechanical time correlation functions of fluctuating forces are actually contained within the influence functional itself, as briefly described in Appendix D. This is a reflection of a deeper structure in the theory that can be manifested in the form of generalized fluctuationdissipation theorems. This aspect of the formalism has been discussed at some length in Ref. 11.

\section{ACKNOWLEDGMENT}

The author is grateful for a number of stimulating discussions with Professor Michael Fayer, Stanford University, and Professor Jim Skinner, University of Wisconsin, during the preparation of an early draft of this article.

\section{APPENDIX A: EVALUATION OF THE INFLUENCE FUNCTIONAL}

Although $F\left[x_{1}, y_{1} ; x_{2}, y_{2}\right]$ as defined by Eq. (16) is not known in general, it can be determined exactly when the coupling between system and bath is linear [corresponding to $n=1$ in Eq. (5)] and when $\rho_{B}\left(R^{\prime}, Q^{\prime}\right)$ describes the density matrix of a system of $N$ independent oscillators,

$$
\begin{aligned}
\rho_{B}\left(R^{\prime}, Q^{\prime}\right)= & \prod_{k=1}^{N}\left(\frac{M_{k} \Omega_{k}}{2 \pi \hbar \sinh \left(\beta \hbar \Omega_{k}\right)}\right)^{1 / 2} \\
& \times \exp \left\{-\frac{M_{k} \Omega_{k}}{2 \hbar \sinh \left(\beta \hbar \Omega_{k}\right)}\left[\left(R_{k}^{\prime 2}+Q_{k}^{\prime 2}\right)\right.\right. \\
& \left.\left.\times \cosh \left(\beta \hbar \Omega_{k}\right)-2 R_{k}^{\prime} Q_{k}^{\prime}\right]\right\} .
\end{aligned}
$$

Here $\beta=1 / k_{B} T$, and a product over the Cartesian components of the vectors is understood. The fact the influence functional is unity when there are no interactions between system and bath (i.e., when $S_{I}=0$ ) suggests treating the nonlinearities in $S_{I}$ (with $n \geqslant 2$ ) as a perturbation around the $n$ $=1$ results. With $\rho_{B}\left(R^{\prime}, Q^{\prime}\right)$ given by Eq. (A1), a perturbation expansion in the nonlinearities then takes the form of a cumulant expansion: ${ }^{12,15}$

$F\left[x_{1}, y_{1} ; x_{2}, y_{2}\right]=\exp \left[\frac{i}{\hbar}\left\langle S_{X}\right\rangle_{0}-\frac{1}{2 \hbar^{2}}\left\{\left\langle S_{X}^{2}\right\rangle_{0}-\left\langle S_{X}\right\rangle_{0}^{2}\right\}+\cdots\right]$,

where $S_{X} \equiv S_{I}\left[x_{1}, x_{2}, R\right]-S_{I}\left[y_{1}, y_{2}, Q\right]$, and the average $\langle(\cdots)\rangle_{0}$ is defined by

$$
\begin{aligned}
\langle(\cdots)\rangle_{0}= & \int d R^{\prime} d Q^{\prime} d R \int_{R^{\prime}}^{R} \mathcal{D}[R] \int_{Q^{\prime}}^{R} \mathcal{D}[Q] \\
& \times \mathrm{e}^{i S_{0} / \hbar}(\cdots) \rho_{B}\left(R^{\prime}, Q^{\prime}\right),
\end{aligned}
$$

with $S_{0} \equiv S_{B}[R]-S_{B}[Q]$. To evaluate the averages in Eq. (A2), which are averages of various powers of the bath normal modes $\{R\}$ and $\{Q\}$, a functional $F[U, V]$ of two fictitious fields $U(\tau)$ and $V(\tau)$ is introduced. This functional is defined by

$$
\begin{aligned}
F[U, V]= & \left\{\prod_{k} \int d R_{k}^{\prime} d Q_{k}^{\prime} d R_{k} \int_{R_{k}^{\prime}}^{R_{k}} \mathcal{D}\left[R_{k}\right] \int_{Q_{k}^{\prime}}^{R_{k}} \mathcal{D}\left[Q_{k}\right]\right\} \\
& \times \mathrm{e}^{i S_{0} / \hbar} \rho_{B}\left(R^{\prime}, Q^{\prime}\right) \exp \left[\frac{i}{\hbar} \sum_{k} \int_{0}^{t} d \tau U_{k}(\tau) R_{k}(\tau)\right. \\
& \left.-\frac{i}{\hbar} \sum_{k} \int_{0}^{t} d \tau V_{k}(\tau) Q_{k}(\tau)\right]
\end{aligned}
$$

which is Eq. (16) with $n=1, \quad B^{(1)}=0, \quad A^{(1)} c_{k} x_{1}(\tau)$ $=-U_{k}(\tau)$, and $A^{(1)} c_{k} y_{1}(\tau)=-V_{k}(\tau)$. The results of Ref. 11 thus allow $F[U, V]$ to be determined at once:

$$
\begin{aligned}
F[U, V]= & \exp \left\{-\sum_{k} d_{k} \int_{0}^{t} d \tau \int_{0}^{t} d \tau^{\prime}\left[U_{k}(\tau)-V_{k}(\tau)\right]\right. \\
& \left.\times\left[\alpha_{k}^{*}\left(\tau-\tau^{\prime}\right) U_{k}\left(\tau^{\prime}\right)-\alpha_{k}\left(\tau-\tau^{\prime}\right) V_{k}\left(\tau^{\prime}\right)\right]\right\},
\end{aligned}
$$

where

$$
d_{k}=\frac{1}{4 M_{k} \Omega_{k} \hbar}
$$


and

$$
\begin{aligned}
\alpha_{k}\left(\tau-\tau^{\prime}\right)= & \frac{1}{1-\mathrm{e}^{-\beta \hbar \Omega_{k}}} \mathrm{e}^{i \Omega_{k}\left(\tau-\tau^{\prime}\right)} \\
& +\frac{\mathrm{e}^{-\beta \hbar \Omega_{k}}}{1-\mathrm{e}^{-\beta \hbar \Omega_{k}}} \mathrm{e}^{-i \Omega_{k}\left(\tau-\tau^{\prime}\right)}
\end{aligned}
$$

with $\alpha_{k}^{*}\left(\tau-\tau^{\prime}\right)$ the complex conjugate of $\alpha_{k}\left(\tau-\tau^{\prime}\right)$. Two other equivalent forms of this function will be used in what follows:

$$
\alpha_{k}\left(\tau-\tau^{\prime}\right)=z_{k} \cos \left(\Omega_{k}\left(\tau-\tau^{\prime}\right)\right)+i \sin \left(\Omega_{k}\left(\tau-\tau^{\prime}\right)\right),
$$

where $z_{k}=\operatorname{coth}\left(\beta \hbar \Omega_{k} / 2\right)$, and

$$
\alpha_{k}\left(\tau-\tau^{\prime}\right)=\left(1+n_{k}\right) \mathrm{e}^{i \Omega_{k}\left(\tau-\tau^{\prime}\right)}+n_{k} \mathrm{e}^{-i \Omega_{k}\left(\tau-\tau^{\prime}\right)},
$$

where $n_{k} \equiv 1 /\left[\exp \left(\beta \hbar \Omega_{k}\right)-1\right]$, the mean thermal occupation number.

Averages of the bath coordinates may now be obtained as functional derivatives of $F[U, V]$ with respect to $U$ or $V$. By way of illustration, one sees from Eq. (A4) that

$$
\begin{aligned}
& \left\langle R_{i}(\tau) R_{j}(\tau) Q_{k}\left(\tau^{\prime}\right) R_{l}\left(\tau^{\prime}\right)\right\rangle_{0} \\
& \quad=\left.\hbar^{4} \frac{\delta^{4} F}{\delta U_{i}(\tau) \delta U_{j}(\tau) \delta V_{k}\left(\tau^{\prime}\right) \delta V_{l}\left(\tau^{\prime}\right)}\right|_{U, V=0} .
\end{aligned}
$$

The functional differentiation of Eq. (A5) according to the prescription above leads to

$$
\begin{aligned}
& \left\langle R_{i}(\tau) R_{j}(\tau) Q_{k}\left(\tau^{\prime}\right) R_{l}\left(\tau^{\prime}\right)\right\rangle_{0} \\
& =\hbar^{4}\left[4 d_{k} d_{l} \alpha_{k}\left(\tau-\tau^{\prime}\right) \alpha_{l}\left(\tau-\tau^{\prime}\right)\left(\delta_{i l} \delta_{j k}+\delta_{i k} \delta_{j l}\right)\right. \\
& \left.\quad+4 \delta_{i j} \delta_{k l} d_{j} d_{l} \alpha_{j}(0) \alpha_{l}(0)\right] .
\end{aligned}
$$

Other averages of the bath coordinates are obtained similarly. (Averages involving combinations of powers of $R_{k}$ and $Q_{k}$ that have an overall odd exponent are 0 by symmetry.) In this way, the quantities $\left\langle S_{X}\right\rangle_{0}$ and $\left\langle S_{X}^{2}\right\rangle_{0}$ are calculated for different values of $n$. The results are then substituted into Eq. (A2) to produce expressions for $F\left[x_{1}, y_{1} ; x_{2}, y_{2}\right]$; these are then used to determine probabilities of transition between different states of the coupled oscillator pair.

\section{APPENDIX B: QUADRATIC NONLINEARITIES IN THE SOLVENT MODES}

When $n=2$, corresponding to a second power of the solvent collective coordinate in the interaction Hamiltonian, it can be shown that $\left\langle S_{X}\right\rangle_{0}$, the mean of the interaction action, is given by

$$
\begin{aligned}
\left\langle S_{X}\right\rangle_{0}= & -2 \hbar^{2} \sum_{n} d_{n} c_{n}^{2} \alpha_{n}(0) \int_{0}^{t} d \tau\left\{A^{(2)}\left[x_{1}(\tau)-y_{1}(\tau)\right]\right. \\
& \left.+B^{(2)}\left[x_{2}(\tau)-y_{2}(\tau)\right]\right\} .
\end{aligned}
$$

Because its dependence on the oscillator coordinates is linear, $\left\langle S_{X}\right\rangle_{0}$ merely renormalizes the potential, ${ }^{15}$ so its contribution to the influence functional is ignored, and only the second cumulant of $S_{X}$ is considered further. Evaluating this quantity by the methods described earlier, and substituting the result into Eq. (A2), one finds that

$$
\begin{aligned}
F\left[x_{1}, y_{1} ; x_{2}, y_{2}\right]= & F_{1}^{(2)}\left[x_{1}, y_{1}\right] F_{2}^{(2)}\left[x_{2}, y_{2}\right] \\
& \times F_{12}^{(2)}\left[x_{1}, y_{1} ; x_{2}, y_{2}\right],
\end{aligned}
$$

where

$$
\begin{aligned}
F_{1}^{(2)}\left[x_{1}, y_{1}\right]= & \exp \left[-4 \hbar^{2}\left(A^{(2)}\right)^{2} \sum_{m, n} d_{m} d_{n} c_{m}^{2} c_{n}^{2}\right. \\
& \times \int_{0}^{t} \int_{0}^{t} d \tau d \tau^{\prime}\left\{\left[x_{1}(\tau)-y_{1}(\tau)\right]\right. \\
& \times R_{m n}^{(2)}\left(\tau, \tau^{\prime}\right)\left[x_{1}\left(\tau^{\prime}\right)-y_{1}\left(\tau^{\prime}\right)\right] \\
& -i\left[x_{1}(\tau)-y_{1}(\tau)\right] \\
& \left.\left.\times I_{m n}^{(2)}\left(\tau, \tau^{\prime}\right)\left[x_{1}\left(\tau^{\prime}\right)+y_{1}\left(\tau^{\prime}\right)\right]\right\}\right],
\end{aligned}
$$

and

$$
\begin{aligned}
& F_{12}^{(2)}\left[x_{1}, y_{1} ; x_{2}, y_{2}\right] \\
& =\exp \left[-8 \hbar^{2} A^{(2)} B^{(2)} \sum_{m, n} d_{m} d_{n} c_{m}^{2} c_{n}^{2}\right. \\
& \quad \times \int_{0}^{t} \int_{0}^{t} d \tau d \tau^{\prime}\left\{\left[x_{1}(\tau)-y_{1}(\tau)\right]\right. \\
& \quad \times R_{m n}^{(2)}\left(\tau, \tau^{\prime}\right)\left[x_{2}\left(\tau^{\prime}\right)-y_{2}\left(\tau^{\prime}\right)\right] \\
& \left.\left.\quad-i\left[x_{1}(\tau)-y_{1}(\tau)\right] I_{m n}^{(2)}\left(\tau, \tau^{\prime}\right)\left[x_{2}\left(\tau^{\prime}\right)+y_{2}\left(\tau^{\prime}\right)\right]\right\}\right]
\end{aligned}
$$

where

$$
\begin{aligned}
R_{m n}^{(2)}\left(\tau, \tau^{\prime}\right)= & \frac{1}{2}\left(z_{m} z_{n}+1\right) \cos \left(\theta_{m}+\theta_{n}\right) \\
& +\frac{1}{2}\left(z_{m} z_{n}-1\right) \cos \left(\theta_{m}-\theta_{n}\right)
\end{aligned}
$$

and

$$
\begin{aligned}
I_{m n}^{(2)}\left(\tau, \tau^{\prime}\right)= & \frac{1}{2}\left(z_{m}+z_{n}\right) \sin \left(\theta_{m}+\theta_{n}\right) \\
& +\frac{1}{2}\left(z_{n}-z_{m}\right) \sin \left(\theta_{m}-\theta_{n}\right)
\end{aligned}
$$

with $\theta_{i}=\Omega_{i}\left(\tau-\tau^{\prime}\right)$ and $z_{i}$ defined after Eq. (A8). The function $F_{2}^{(2)}\left[x_{2}, y_{2}\right]$ is obtained from $F_{1}^{(2)}\left[x_{1}, y_{1}\right]$ by replacing $A^{(2)}$ by $B^{(2)}, x_{1}$ by $x_{2}$ and $y_{1}$ by $y_{2}$.

\section{APPENDIX C: CUBIC NONLINEARITIES IN THE SOLVENT MODES}

Here $n=3$, corresponding to a third power of the solvent collective coordinate in the interaction Hamiltonian. For this case, the influence functional is found to be

$$
\begin{aligned}
F\left[x_{1}, y_{1} ; x_{2}, y_{2}\right]= & F_{1}^{(3)}\left[x_{1}, y_{1}\right] F_{2}^{(3)}\left[x_{2}, y_{2}\right] \\
& \times F_{12}^{(3)}\left[x_{1}, y_{1} ; x_{2}, y_{2}\right],
\end{aligned}
$$

where 


$$
\begin{aligned}
F_{1}^{(3)}\left[x_{1}, y_{1}\right]= & \exp \left[-24 \hbar^{4}\left(A^{(3)}\right)^{2} \sum_{l, m, n} d_{l} d_{m} d_{n} c_{l}^{2} c_{m}^{2} c_{n}^{2} \int_{0}^{t} \int_{0}^{t} d \tau d \tau^{\prime}\left\{\left[x_{1}(\tau)-y_{1}(\tau)\right]\right.\right. \\
& \left.\left.\times R_{l m n}^{(3)}\left(\tau, \tau^{\prime}\right)\left[x_{1}\left(\tau^{\prime}\right)-y_{1}\left(\tau^{\prime}\right)\right]-i\left[x_{1}(\tau)-y_{1}(\tau)\right] I_{l m n}^{(3)}\left(\tau, \tau^{\prime}\right)\left[x_{1}\left(\tau^{\prime}\right)+y_{1}\left(\tau^{\prime}\right)\right]\right\}\right]
\end{aligned}
$$

and

$$
\begin{aligned}
F_{12}^{(3)}\left[x_{1}, y_{1} ; x_{2}, y_{2}\right]= & \exp \left[-48 \hbar^{4} A^{(3)} B^{(3)} \sum_{l, m, n} d_{l} d_{m} d_{n} c_{l}^{2} c_{m}^{2} c_{n}^{2} \int_{0}^{t} \int_{0}^{t} d \tau d \tau^{\prime}\left\{\left[x_{1}(\tau)-y_{1}(\tau)\right]\right.\right. \\
& \left.\left.\times R_{l m n}^{(3)}\left(\tau, \tau^{\prime}\right)\left[x_{2}\left(\tau^{\prime}\right)-y_{2}\left(\tau^{\prime}\right)\right]-i\left[x_{1}(\tau)-y_{1}(\tau)\right] I_{l m n}^{(3)}\left(\tau, \tau^{\prime}\right)\left[x_{2}\left(\tau^{\prime}\right)+y_{2}\left(\tau^{\prime}\right)\right]\right\}\right],
\end{aligned}
$$

where

$$
\begin{aligned}
R_{l m n}^{(3)}\left(\tau, \tau^{\prime}\right)= & \frac{1}{4}\left[\left(z_{l} z_{m} z_{n}+z_{l}+z_{m}+z_{n}\right) \cos \left(\theta_{l}+\theta_{m}+\theta_{n}\right)+\left(z_{l} z_{m} z_{n}-z_{l}-z_{m}+z_{n}\right) \cos \left(\theta_{l}+\theta_{m}-\theta_{n}\right)\right. \\
& \left.+\left(z_{l} z_{m} z_{n}-z_{l}+z_{m}-z_{n}\right) \cos \left(\theta_{l}-\theta_{m}+\theta_{n}\right)+\left(z_{l} z_{m} z_{n}+z_{l}-z_{m}-z_{n}\right) \cos \left(\theta_{l}-\theta_{m}-\theta_{n}\right)\right]+\frac{3}{2} z_{l} z_{m} z_{n} \cos \theta_{n}
\end{aligned}
$$

and

$$
\begin{aligned}
I_{l m n}^{(3)}\left(\tau, \tau^{\prime}\right)= & \frac{1}{4}\left[\left(z_{l} z_{m}+z_{l} z_{n}+z_{m} z_{n}+1\right) \sin \left(\theta_{l}+\theta_{m}+\theta_{n}\right)-\left(z_{l} z_{m}-z_{l} z_{n}-z_{m} z_{n}+1\right) \sin \left(\theta_{l}+\theta_{m}-\theta_{n}\right)\right. \\
& \left.+\left(z_{l} z_{m}-z_{l} z_{n}+z_{m} z_{n}-1\right) \sin \left(\theta_{l}-\theta_{m}+\theta_{n}\right)-\left(z_{l} z_{m}+z_{l} z_{n}-z_{m} z_{n}-1\right) \sin \left(\theta_{l}-\theta_{m}-\theta_{n}\right)\right]+\frac{3}{2} z_{l} z_{m} \sin \theta_{n}
\end{aligned}
$$

with $\theta_{m}=\Omega_{m}\left(\tau-\tau^{\prime}\right)$ as before.

\section{APPENDIX D: FORCE CORRELATION FUNCTIONS FROM THE INFLUENCE FUNCTIONAL}

Expressions for the friction coefficients may be obtained directly from the expressions for the influence functionals themselves. To see how this comes about, notice that had the interactions between the displacements of the solute oscillators and the medium been described in terms of a fluctuating force $f$ rather than in terms of a collection of $N$ other independent oscillators, Eq. (5) would have been given by

$$
H_{X B}=a x_{1} f+b x_{2} f,
$$

where $a$ and $b$ are constants, and the resulting influence functional $F^{\prime}\left[x_{1}, y_{1} ; x_{2}, y_{2}\right]$ would have assumed the form

$$
\begin{aligned}
F^{\prime}\left[x_{1}, y_{1} ; x_{2}, y_{2}\right]= & \exp \left[\frac { i } { \hbar } \int _ { 0 } ^ { t } d \tau \left\{a f(\tau)\left[x_{1}(\tau)-y_{1}(\tau)\right]\right.\right. \\
& \left.\left.+b f(\tau)\left[x_{2}(\tau)-y_{2}(\tau)\right]\right\}\right]
\end{aligned}
$$

Further, if the fluctuations in $f$ were governed by a Guassian probability distribution, such that

$$
\left\langle f(\tau) f\left(\tau^{\prime}\right)\right\rangle=\phi\left(\tau-\tau^{\prime}\right),
$$

$\phi$ being some known function of its arguments, the force averaged influence functional $\bar{F}^{\prime}\left[x_{1}, y_{1} ; x_{2}, y_{2}\right]$ would be

$$
\begin{aligned}
\bar{F}^{\prime}\left[x_{1}, x_{2} ; y_{1}, y_{2}\right] \\
=\exp \left[-\frac{1}{2 \hbar^{2}} \int_{0}^{t} \int_{0}^{t} d \tau d \tau^{\prime}\left\{a^{2}\left[x_{1}(\tau)-y_{1}(\tau)\right] \phi\left(\tau-\tau^{\prime}\right)\right.\right. \\
\quad \times\left[x_{1}\left(\tau^{\prime}\right)-y_{1}\left(\tau^{\prime}\right)\right]+b^{2}\left[x_{2}(\tau)-y_{2}(\tau)\right] \phi\left(\tau-\tau^{\prime}\right) \\
\quad \times\left[x_{2}\left(\tau^{\prime}\right)-y_{2}\left(\tau^{\prime}\right)\right]+2 a b\left[x_{1}(\tau)-y_{1}(\tau)\right] \\
\left.\left.\quad \times \phi\left(\tau-\tau^{\prime}\right)\left[x_{2}\left(\tau^{\prime}\right)-y_{2}\left(\tau^{\prime}\right)\right]\right\}\right]
\end{aligned}
$$

Aside from the absence of purely imaginary contributions, this functional is very similar in structure to the functionals that describe quadratic and cubic nonlinearities in the interactions between solute and solvent [Eqs. (B4) and (C3)]. Caldeira and Leggett ${ }^{21}$ argued on the basis of the analogous correspondence that obtained for the case of linear solutesolvent interactions that the real part of the the modedependent portion of the influence functional could be identified with a symmetrized, quantum mechanical time correlation function of fluctuating forces. As is easily verified from an inspection of the first terms of Eqs. (B5) and (C4) (i.e., the first terms of the real portions $R_{m n}^{(2)}$ and $R_{l m n}^{(3)}$ ), such an identification leads directly to Eqs. (47) and (52) once the phenomenological coefficients $a$ and $b$ that appear in Eq. (D1) are suitably adjusted.

\footnotetext{
${ }^{1}$ J. S. Bader and B. J. Berne, J. Chem. Phys. 100, 8359 (1994).

${ }^{2}$ J. L. Skinner, J. Chem. Phys. 107, 8717 (1997).

${ }^{3}$ S. A. Egorov and B. J. Berne, J. Chem. Phys. 107, 6050 (1997).

${ }^{4}$ P. Schofield, Phys. Rev. Lett. 4, 239 (1960).

${ }^{5}$ P. A. Egelstaff, Adv. Phys. 11, 203 (1962).
} 
${ }^{6}$ L. Frommhold, Collision-Induced Absorption in Gases (Cambridge University Press, Cambridge, 1993).

${ }^{7}$ A. Nitzan and J. Jortner, Mol. Phys. 25, 713 (1973).

${ }^{8}$ A. Nitzan, S. Mukamel, and J. Jortner, J. Chem. Phys. 60, 3929 (1974).

${ }^{9}$ D. Rostkier-Edelstein, P. Graf, and A. Nitzan, J. Chem. Phys. 107, 10470 (1997).

${ }^{10}$ R. Karrlein and H. Grabert, J. Chem. Phys. 108, 4972 (1998).

${ }^{11}$ R. P. Feynman and F. L. Vernon, Ann. Phys. 24, 118 (1963); R. P. Feynman and A. R. Hibbs, Quantum Mechanics and Path Integrals (McGraw-Hill, New York, 1965).

${ }^{12}$ M. Shiga and S. Okazaki, J. Chem. Phys. 109, 3542 (1998).

${ }^{13}$ S. A. Egorov and J. L. Skinner, J. Chem. Phys. 103, 1533 (1995).

${ }^{14}$ D. J. Myers, M. Shigeiwa, M. D. Fayer, and B. J. Cherayil, J. Phys. Chem. B 104, 2402 (2000); D. J. Myers, S. Chen, M. Shigeiwa, B. J. Cherayil, and M. D. Fayer, J. Chem. Phys. 109, 5971 (1998); R. S. Urdahl, K. D. Rector, D. J. Myers, P. H. Davis, and M. D. Fayer, ibid. 105, 8973 (1996); A. Tokmakoff, B. Sauter, and M. D. Fayer, ibid. 100, 9035 (1994); J. D. Beckerle, M. P. Casassa, R. R. Cavanagh, E. J. Heilweil, and J. C. Stephenson, Chem. Phys. 160, 487 (1992).
${ }^{15}$ B. L. Hu, J. P. Paz, and Y. Zhang, Phys. Rev. D 45, 2843 (1992); 47, 1576 (1993).

${ }^{16}$ W. H. Zurek, Phys. Today 44, 36 (1991).

${ }^{17}$ N. Makri, J. Phys. Chem. B 103, 2823 (1999); J. Shao and N. Makri, Phys. Rev. E 59, 269 (1999); J. Ray and N. Makri, J. Phys. Chem. A 103, 9417 (1999).

${ }^{18}$ L. Landau and E. Teller, Phys. Z. Sowjetunion 10, 34 (1936).

${ }^{19}$ D. W. Oxtoby, Adv. Chem. Phys. 40, 1 (1979); J. L. Skinner and D. Hsu, J. Phys. Chem. 90, 4931 (1986).

${ }^{20}$ K. S. Schweizer and D. Chandler, J. Chem. Phys. 76, 2296 (1982); R. B. Williams and R. F. Loring, ibid. 110, 10899 (1999).

${ }^{21}$ A. O. Caldeira and A. J. Leggett, Physica A 121, 587 (1983); A. J. Leggett, S. Chakravarty, A. J. Dorsey, M. P. A. Fisher, A. Garg, and W. Zwerger, Rev. Mod. Phys. 59, 1 (1987); H. Grabert, P. Schramm, and G.-L. Ingold, Phys. Rep. 168, 115 (1988).

${ }^{22}$ V. M. Kenkre, A. Tokmakoff, and M. D. Fayer, J. Chem. Phys. 101, 10618 (1994). 